\title{
ON CERTAIN CLASSES OF VARIATIONAL INEQUALITIES AND RELATED ITERATIVE ALGORITHMS
}

\author{
MUHAMMAD ASLAM NOOR \\ King Saud University, Mathematics Department \\ College of Science, P.O. Box 2455, Riyadh 11451 \\ Saudi Arabia \\ E-mail: F40M040@SAKSU00.BITNET
}

(Received October, 1994; Revised October, 1995)

\begin{abstract}
In this paper, we introduce and study some new classes of variational inequalities and Wiener-Hopf equations. Essentially using the projection technique, we establish the equivalence between the multivalued general quasi-variational inequalities and the multivalued implicit Wiener-Hopf equations. This equivalence enables us to suggest and analyze a number of iterative algorithms for solving multivalued general quasi-variational inequalities. We also consider the auxiliary principle technique to prove the existence of a unique solution of the variationallike inequalities. This technique is used to suggest a general and unified iterative algorithm for computing the approximate solution. Several special cases which can be obtained from our main results are also discussed. The results proved in this paper represent a significant refinement and improvement of the previously known results.
\end{abstract}

Key words: Variational Inequalities, Wiener-Hopf Equations, Auxiliary Principle, Iterative Algorithm, Convergence Analysis.

AMS (MOS) subject classifications: 49J40, 90C20.

\section{Introduction}

The theory of variational inequalities emerged as an interesting and fascinating branch of applicable mathematics. This theory constituted an important and significant extension of the variational principles and led to innovative applications in diverse fields. In fact, the variational inequality theory provides us a natural, simple, general, and unified framework to study a wide class of unrelated linear and nonlinear problems arising in fluid mechanics, elasticity, and oceanography. Furthermore, the variational inequality theory provides us this very same framework to study general equilibrium problems in economics and transportation, optimization, operations research, and physical, regional, and engineering sciences; see, for example, [1-35] and the references therein. In recent years, various extensions and generalizations of the variational inequalities have been proposed and analyzed using new ideas and techniques. It is worth mentioning that the theory of variational inequalities can be considered as the natural development of the 19th and 20th problems of Hilbert, which he formulated in his famous Paris lecture in 1900. This theory allows us to prove not only the existence of solutions for nonlinear equations and inequalities (which are not necessarily the Euler-Lagrange equations corresponding to variational pro- 
blems), but also helps us in developing efficient numerical techniques for solving them.

Inspired and motivated by the recent research work going on in this field, we introduce and consider some new classes of variational inequalities. Noor [25] studied the multivalued general quasi-variational inequalities, where the projection method was used to suggest an iterative algorithm for finding the approximate solution. In this paper, we prove that the multivalued general quasi-variational inequality is equivalent to a system of equations, known as the multivalued implicit Wiener-Hopf equations. This equivalence is quite flexible and general. By a suitable rearrangement of the implicit Wiener-Hopf equations, we suggest a number of iterative algorithms for solving quasi-variational inequalities. We also consider another new class of variational inequalities, which is known as the strongly nonlinear mixed variational-like inequality. This class has important and potential applications in economics and transportation equilibrium problems, optimization theory, and nonlinear analysis. We note that the projection method and its variant forms cannot be used to study the existence of the solution of these mixed variational-like inequalities, since it is not possible to obtain the projection of the space into the product of the convex sets. This fact motivates us to use the auxiliary principle technique of Glowinski, Lions, and Tremolieres [10] and Noor [18-23] to study the existence of a solution of the mixed variational-like inequalities. This technique deals with the auxiliary variational-like inequality and proves that the solution of the auxiliary problem is the solution of the original variational-like inequality. In addition, this technique can be used to suggest a novel and general iterative algorithm. Recently, it has been shown that the auxiliary principle technique helps us in finding the appropriate variational principles for different classes of variational inequalities, which provide us with a general framework to analyze and describe many computational algorithms ranging from gradient, Newton, decomposition, and descent algorithms; see Zhu and Marcotte [35], Noor [23], and Larsson and Patriksson [14] for more details.

In Section 2, we formulate the problems and discuss some special cases which can be derived from these new problems. In Section 3, we establish the equivalence between the multivalued general quasi variational inequalities and the multivalued implicit Wiener-Hopf equations. This equivalence is used to analyze some iterative algorithms for variational inequalities. The auxiliary principle technique is used to prove the existence of a unique solution of variational-like inequalities, which is the subject of Section 4. We show that the auxiliary principle technique can be used to suggest a general iterative algorithm for variational inequalities.

\section{Preliminaries}

Let $H$ be a real Hilbert space whose norm and inner product are denoted by $\|\cdot\|$ and $\langle\cdot, \cdot\rangle$, respectively. Let $K$ be a nonempty closed convex set in $H$. Let $T, A, g: H \rightarrow H$ be single-valued operators and $V: H \rightarrow 2^{H}$ be a multivalued operator.

Given a point-to-set mapping $K: u \rightarrow K(u)$, which associates a closed convex set $K(u)$ with any element $u$ of $H$, consider the problem of finding $u \in H$ and $w \in V(u)$ such that $g(u) \in K(u)$ and

$$
\langle T u+A w, g(v)-g(u)\rangle \geq 0, \text { for all } g(v) \in K(u) .
$$

The problem of type (2.1) is called the multivalued general nonlinear quasi-variational inequality problem. This problem is mainly and originally due to Noor [25], where the projection method was used to study the existence of a solution of the multivalued quasi-variational inequalities (2.1). It has been shown in [25] that implicit obstacle odd order boundary value problems can be studied in the general framework of quasi-variational inequalities (2.1). 
Related to multivalued general quasi variational inequality (2.1), we consider the problem of solving the generalized implicit Wiener-Hopf equations. Let $P_{K(u)}$ be the projection of $H$ into $K(u)$ and $Q_{K(u)} \equiv I-P_{k(u)}$, where $I$ is the identity operator. We consider the problem of finding $z \in H, u \in H$ and $w \in V(u)$ such that

$$
T g^{-1} P_{K(u)} z+\rho^{-1} Q_{K(u)} z=-A(w)
$$

where $\rho>0$ is a constant and $g^{-1}$ is the inverse of the operator $g$. Equations of type (2.2) are called multivalued implicit Wiener-Hopf equations. For the general treatment, formulations, and applications, see Speck [31], Shi [30], and Noor-Noor-Rassias [27].

Given single-valued nonlinear operators $T, A: H \rightarrow H$ and $\eta: H \times H \rightarrow H$, we consider the problems of finding $u \in H$ such that $\eta(u, v) \in K \times K$ and

$$
\langle T u+A(u), \eta(v, u)\rangle+j(v)-j(u) \geq 0, \text { for all } v \in H
$$

where $j: H \rightarrow H$ is a proper, semi-continuous, convex and nondifferentiable function. This problem is called the strongly nonlinear mixed variational-like inequality problem. It has many applications in optimization theory, engineering sciences, economics, calculus of variations, and network equilibrium; see, for example, [19,21,33,34].

Special Cases: I. If $\eta(v, u)=v-u$, then problem (2.3) is equivalent to finding $u \in H$ such that

$$
\langle T u+A(u), v-u\rangle+j(v)-j(u) \geq 0, \quad \text { for all } v \in H .
$$

Problem (2.4) is mainly due to Noor [16]. For the applications and numerical methods, see Noor $[16,20]$.

II. If $\eta(v, u)=g(v)-g(u)$ where $g: H \rightarrow H$ is a nonlinear operator, then problem (2.3) is equivalent to finding $u \in H$ such that $g(u) \in K$ and

$$
\langle T u+A(u), g(v)-g(u)\rangle+j(v)-j(u) \geq 0, \text { for all } g(v) \in K,
$$

and is known as the general strongly nonlinear mixed variational inequality problem [20].

III. If $A(u) \equiv 0$, then problem (2.3) is equivalent to finding $u \in H$ such that

$$
\langle T u, \eta(v, u)\rangle+j(v)-j(u) \geq 0, \text { for all } v \in H,
$$

which is a problem considered and studied by Noor [19], where the auxiliary principle technique was used to study the existence of a unique solution and to suggest an iterative algorithm.

IV. If $j(u) \equiv 0$, then problem (2.3) reduces to finding $u \in H$ such that $\eta(u, v) \in K \times K$ and

$$
\langle T u+A(u), \eta(v, u)\rangle \geq 0, \text { for all } v \in H .
$$

Inequality (2.7) is called the strongly nonlinear variational-like inequality, which is mainly due to Noor [21].

In brief, for a suitable choice of operators $T, A, g, V, \eta$, and convex set $K(u)$, one can arrive at various classes of variational inequalities, complementarity problems, and the Wiener-Hopf equations as special cases of problems (2.1) - (2.7).

We need the following concepts and results. 
Definition 2.1: The nonlinear operator $\eta: H \times H \rightarrow H$ is said to be

(a) Strongly monotone if there exists a constant $\alpha>0$ such that

$$
\langle\eta(v, u), v-u\rangle \geq \alpha\|v-u\|^{2}, \text { for all } u, v \in H .
$$

(b) Lipschitz continuous if there exists a constant $\beta>0$ such that

$$
\|\eta(v, u)\| \leq \beta\|v-u\|, \text { for all } u, v \in H
$$

We would like to point out that if $\eta(v, u)=T v-T u$, where $T: H \rightarrow H$ is a single-valued operator, then definition 2.1 reduces to the usual definition of strong monotonicity and Lipschitz continuity of nonlinear operator $T$. From $(a)$ and $(b)$, it follows that $\alpha \leq \beta$. Note that if $\beta=1$, then the operator $\eta$ is nonexpansive.

Definition 2.2: The multivalued mapping $V: H \rightarrow C(H)$ is called M-Lipschitz continuous if there exists a constant $\mu>0$ such that

$$
M(V(u), V(v)) \leq \mu\|u-v\|, \text { for all } u, v \in H,
$$

where $C(H)$ is the family of all nonempty compact subsets of $H$ and $M(\cdot, \cdot)$ is the Hausdorff metric on $C(H)$.

Assumption 2.1: The nonlinear operator $\eta: H \times H \rightarrow H$ satisfies the relation

$$
\eta(v, u)=-\eta(u, v), \text { for all } u, v \in H
$$

Obviously $\eta(u, v)=0$, for all $u \in H$, and it was used in [34,35] to study the existence of a solution of the variational-like inequalities.

Lemma 2.1 [1]: Let $K$ be a closed convex set in $H$. Then, for a given $z \in H, u=P_{K} z$ if and only if $u \in K$ satisfies

$$
\langle u-z, v-u\rangle \geq 0 \text { for all } v \in K
$$

Furthermore, the projection operator $P_{K}$ is nonexpansive, that is,

$$
\left\|P_{K} u-P_{K} v\right\| \leq\|u-v\|, \text { for all } u, v \in H \text {. }
$$

\section{Wiener-Hopf Equations Technique}

First of all, we prove the equivalence between the multivalued general quasi-variational inequality (2.1) and the multivalued implied Wiener-Hopf equation (2.2) essentially using the projection technique of Noor $[17,24]$ and Shi [30].

Theorem 3.1: The multivalued general quasi-variational inequality (2.1) has a solution $u \in H, w \in V(u)$ if and only if the multivalued implicit Wiener-Hopf equation (2.2) has a solution $z \in H, u \in H, w \in V(u)$, where

$$
g(u)=P_{K(u)^{z}},
$$




$$
z=g(u)-\rho(T u+A w)
$$

and $\rho>0$ is a constant.

Proof: Let $u \in H, w \in V(u)$ such that $g(u) \in K(u)$ be a solution of the variational inequality (2.1). Then, by Lemma 2.1 , we have

$$
g(u)=P_{K(u)}[g(u)-\rho(T u+A w)]
$$

Now, using the fact that $Q_{K(u)}=I-P_{K(u)}$ and equation (3.3), we obtain

$$
\begin{gathered}
Q_{K(u)}[g(u)-\rho(T u+A w)]=g(u)-\rho(T u+A w)-P_{K(u)}[g(u)-\rho(T u+A w)] \\
=-\rho(T u+A w) \\
=-\rho T g^{-1} P_{K(u)}[g(u)-\rho(T u+A w)]-\rho A w
\end{gathered}
$$

from which and (3.2), it follows that

$$
T g^{-1} P_{K(u)} z+\rho^{-1} Q_{K(u)} z=-A w .
$$

Conversely, let $z \in H, u \in H, w \in V(u)$ be a solution of $(2.2)$, then

$$
\rho\left(T g^{-1} P_{K(u)} z+A w\right)=-Q_{K(u)} z=P_{K(u)} z-z .
$$

Now from Lemma 2.1 and (3.4), for all $g(v) \in K$, we have

$$
0 \leq\left\langle P_{K(u)} z-z, g(v)-P_{K(u)} z\right\rangle=\rho\left\langle T g^{-1} P_{K(u)} z+A w, g(v)-P_{K(u)} z\right\rangle .
$$

Thus $(u, w)$, where $u=g^{-1} P_{K(u)} z$ is a solution of (2.1).

Theorem 3.1 establishes the equivalence between the variational inequality (2.1) and the Wiener-Hopf equations (2.2). This equivalence is quite general and flexible. For the appropriate rearrangement of the Wiener-Hopf equations (2.2), we can suggest a number of iterative algorithms for solving quasi-variational inequalities (2.1).

I. Equations (2.2) can be written as

$$
Q_{K(u)} z=-\rho\left(T g^{-1} P_{K(u)} z+A w\right)
$$

from which it follows that

$$
\begin{aligned}
z & =P_{K(u)^{z}-\rho T g^{-1} P_{K(u)} z-\rho A w} \\
& =g(u)-\rho T u-\rho A w, \text { using (3.1). }
\end{aligned}
$$

This formulation enables us to suggest the following iterative algorithm for solving the quasivariational inequality $(2.1)$ :

Algorithm 3.1: Assume that $K(u)$ is a closed convex set in $H$. Let $T, A, g: H \rightarrow H$ be nonlinear operators and $V: H \rightarrow C(H)$ be a set-valued mapping. For given $z_{0} \in H$ and $u_{0} \in H$, let us take $w_{0} \in V\left(u_{0}\right), g\left(u_{0}\right) \in K$ and 


$$
\begin{gathered}
g\left(u_{0}\right)=P_{K\left(u_{0}\right)} z_{0} \\
z_{1}=g\left(u_{0}\right)-\rho\left(T u_{0}+A w_{0}\right) .
\end{gathered}
$$

Since $w_{0} \in V\left(u_{0}\right) \in C(H)$, there exists a $w_{1} \in V\left(u_{1}\right)$ such that

$$
\left\|w_{0}-w_{1}\right\| \leq M\left(V\left(u_{0}\right), V\left(u_{1}\right)\right)
$$

where $M(\cdot, \cdot)$ is the Hausdorff metric on $C(H)$. Let

$$
\begin{gathered}
g\left(u_{1}\right)=P_{K\left(u_{1}\right)} z_{1} \\
z_{2}=g\left(u_{1}\right)-\rho\left(T u_{1}+A w_{1}\right) .
\end{gathered}
$$

By induction, we can obtain the sequences $\left\{z_{n}\right\},\left\{u_{n}\right\}$, and $\left\{w_{n}\right\}$ such that

$$
\begin{gathered}
\left.g\left(u_{n}\right)=P_{K\left(u_{n}\right)}\right)^{z} \\
w_{n} \in V\left(u_{n}\right):\left\|w_{n+1}-w_{n}\right\| \leq M\left(V\left(u_{n+1}\right), V\left(u_{n}\right)\right) \\
z_{n+1}=g\left(u_{n}\right)-\rho\left(T u_{n}+A w_{n}\right), \quad n=0,1,2, \ldots
\end{gathered}
$$

II. Equation (2.2) may be written as

$$
Q_{K(u)} z=\left(I-\rho^{-1}\right) Q_{K(u)} z-T g^{-1} P_{K(u)^{z}}-A w
$$

which implies that

$$
\begin{aligned}
& z=P_{K(u)}-T g^{-1} P_{K(u)} z+\left(I-\rho^{-1}\right) Q_{K(u)} z-A w \\
& =g(u)-T u-A w+\left(I-\rho^{-1}\right) Q_{K(u)} z, \quad \text { using (3.1). }
\end{aligned}
$$

Using this fixed point formulation, we can suggest the following iterative scheme:

Algorithm 3.2: For given $z_{0} \in H, u_{0} \in H$, and $w_{0} \in V\left(u_{0}\right)$, compute $\left\{z_{n}\right\},\left\{u_{n}\right\}$, and $\left\{w_{n}\right\}$ from the iterative schemes.

$$
\begin{gathered}
g\left(u_{n}\right)=P_{K(u)^{z}} \\
w_{n} \in V\left(u_{n}\right):\left\|w_{n+1}-w_{n}\right\| \leq M\left(V\left(u_{n+1}\right), V\left(u_{n}\right)\right) \\
\left.z_{n+1}=g\left(u_{n}\right)-\left(T u_{n}+A w_{n}\right)+\left(I-\rho^{-1}\right) Q_{K\left(u_{n}\right.}\right)^{z_{n}}, \quad n=0,1,2, \ldots
\end{gathered}
$$

III. If $T^{-1}$ exists, then equation (2.2) may be written as

$$
P_{K(u)} z=-\rho^{-1} g T^{-1} Q_{K(u)} z-g T^{-1} A w,
$$

from which it follows that

$$
z=\left(I-\rho^{-1} g T^{-1}\right) Q_{K(u)^{z}}-g T^{-1} A w
$$


This fixed point formulation can be used to suggest the following iterative scheme:

Algorithm 3.3: For given $z_{0} \in H, u_{0} \in H$, and $w_{0} \in V\left(u_{0}\right)$, compute $\left\{z_{n}\right\},\left\{u_{n}\right\}$, and $\left\{w_{n}\right\}$ by the iterative schemes

$$
\begin{gathered}
g\left(u_{n}\right)=P_{K\left(u_{n}\right)} z_{n} \\
w_{n} \in V\left(u_{n}\right):\left\|w_{n+1}-w_{n}\right\| \leq M\left(V\left(u_{n+1}\right), V\left(u_{n}\right)\right) \\
\left.z_{n+1}=\left(I-\rho^{-1} g T^{-1}\right) Q_{K\left(u_{n}\right.}\right)^{z_{n}}-g T^{-1} A w_{n}, \quad n=0,1,2, \ldots
\end{gathered}
$$

For a suitable choice of operators $T, g, A, V$, and convex set $K$, one can obtain a number of iterative algorithms for solving various classes of variational inequalities and related complementarity problems.

We now study the convergence criteria of Algorithm 3.1. In a similar way, one can study the convergence of the approximate solutions obtained from Algorithms 3.2 and 3.3.

Concerning the convergence analysis of Algorithm 3.1, we need the following condition:

Assumption 3.1: For all $u, v, z \in H$, the projection operator $P_{K(u)}$ satisfies the condition

$$
\left\|P_{K(u)} z-P_{K(v)} z\right\| \leq \gamma\|u-v\|
$$

where $\gamma>0$ is a constant.

Remark 3.1: We observe that Assumption 3.1 holds true if

$$
K(u)=m(u)+K
$$

where $m$ is a point-to-point mapping and $K$ is a closed convex set in $H$. It is well known that

$$
P_{K(u)}=P_{K+m(u)} v=m(u)+P_{K}[v-m(u)] .
$$

Thus, from the above relations, we have

$$
\begin{gathered}
\left\|P_{K(u)} z-P_{K(v)} z\right\|=\left\|m(u)-m(v)+P_{K}[z-m(u)]-P_{K}[z-m(v)]\right\| \\
\leq\|m(u)-m(v)\|+\left\|P_{K}[z-m(u)]-P_{K}[z-m(v)]\right\| \\
\leq 2\|m(u)-m(v)\| \leq 2 \nu\|u-v\|
\end{gathered}
$$

which implies Assumption 3.1 holds with $\gamma=2 \nu>0$, where $\nu>0$ is the Lipschitz continuity constant of the point-to-point mapping $m$.

Theorem 3.2: Let the operators $T, g: H \rightarrow H$ be strongly monotone with constants $\alpha>0$ and $\sigma>0$, respectively, as well as Lipschitz continuous with constants $\beta>0$ and $\delta>0$ respectively. Let the operator $A: H \rightarrow H$ be Lipschitz continuous with constant $\xi>0$, and $V: H \rightarrow C(H)$ be $M$ Lipschitz continuous with constant $\mu>0$. Suppose that Assumption 3.1 holds and

$$
\left|\rho-\frac{\alpha-\mu \xi(1-k)}{\beta^{2}-\mu^{2} \xi^{2}}\right|<\frac{\sqrt{[\alpha-\mu \xi(1-k)]^{2}-\left(\beta^{2}-\mu^{2} \xi^{2}\right) k(2-k)}}{\beta^{2}-\mu^{2} \xi^{2}}
$$




$$
\begin{gathered}
\alpha>\mu \xi(1-k)+\sqrt{\left(\beta^{2}-\eta^{2} \xi^{2}\right) k(2-k)} \\
\rho \mu \xi<1-k, \\
\text { with } k=2 \sqrt{1-2 \sigma+\delta^{2}}+\gamma .
\end{gathered}
$$

Then there exist $z \in H, u \in H$, and $w \in V(u)$, which are a solution of the multivalued implicit Wiener-Hopf equation (2.2), and the sequences $\left\{z_{n}\right\},\left\{u_{n}\right\}$, and $\left\{w_{n}\right\}$, generated by Algorithm 3.1, converge strongly to $z, u$, and $w$ in $H$, respectively.

Proof: From Algorithm 3.1, we have

$$
\begin{gathered}
\left\|z_{n+1}-z_{n}\right\|=\left\|g\left(u_{n}\right)-g\left(u_{n-1}\right)-\rho\left(T u_{n}-T u_{n-1}\right)-\rho\left(A w_{n}-A w_{n-1}\right)\right\| \\
\leq\left\|u_{n}-u_{n-1}-\left(g\left(u_{n}\right)-g\left(u_{n-1}\right)\right)\right\|+\left\|u_{n}-u_{n-1}-\rho\left(T u_{n}-T u_{n-1}\right)\right\| \\
+\rho\left\|A w_{n}-A w_{n-1}\right\| .
\end{gathered}
$$

Since $T$ is strongly monotone and Lipschitz continuous, therefore

$$
\begin{gathered}
\left\|u_{n}-u_{n-1}-\rho\left(T u_{n}-T u_{n-1}\right)\right\|^{2} \\
=\left\|u_{n}-u_{n-1}\right\|^{2}-2 \rho\left\langle T u_{n}-T u_{n-1}, u_{n}-u_{n-1}\right\rangle+\rho^{2}\left\|T u_{n}-T u_{n-1}\right\|^{2} \\
\leq\left(1-2 \rho \alpha+\beta^{2} \rho^{2}\right)\left\|u_{n}-u_{n-1}\right\|^{2}
\end{gathered}
$$

By using the strong monotonicity and Lipschitz continuity of $g$, we have

$$
\left\|u_{n}-u_{n-1}-\left(g\left(u_{n}\right)-g\left(u_{n-1}\right)\right)\right\|^{2} \leq\left(1-2 \sigma+\delta^{2}\right)\left\|u_{n}-u_{n-1}\right\|^{2} .
$$

Furthermore, since $A$ is Lipschitz continuous and $V$ is $M$-Lipschitz continuous, we have

$$
\begin{gathered}
\left\|A w_{n}-A w_{n-1}\right\| \leq \xi\left\|w_{n}-w_{n-1}\right\| \leq \xi M\left(V\left(u_{n}\right), V\left(u_{n-1}\right)\right) \\
\leq \xi \mu\left\|u_{n}-u_{n-1}\right\|
\end{gathered}
$$

From (3.12)-(3.16), we obtain

$$
\begin{gathered}
\left\|z_{n+1}-z_{n}\right\| \leq\left\{\sqrt{1-2 \sigma+\delta^{2}}+\sqrt{1-2 \rho \alpha+\rho^{2} \beta^{2}}+\rho \xi \mu\right\}\left\|u_{n}-u_{n-1}\right\| \\
=\left\{\frac{k-\gamma}{2}+\sqrt{1-2 \rho \alpha+\rho^{2} \beta^{2}}+\rho \xi \mu\right\}\left\|u_{n}-u_{n-1}\right\| .
\end{gathered}
$$

Now, from (3.6), (3.15), (3.12), and Assumption 3.1, we have

$$
\begin{gathered}
\left\|u_{n}-u_{n-1}\right\| \leq\left\|u_{n}-u_{n-1}-\left(g\left(u_{n}\right)-g\left(u_{n-1}\right)\right)\right\|+\left\|P_{K\left(u_{n}\right)} z_{n}-P_{K\left(u_{n-1}\right)^{z-1}}\right\| \\
\left.\leq \frac{k-\gamma}{2}\left\|u_{n}-u_{n-1}\right\|+\left\|P_{K\left(u_{n}\right)^{z}}-P_{K\left(u_{n}\right)} z_{n-1}\right\|+\| P_{K\left(u_{n}\right)}\right)_{n-1}-P_{K\left(u_{n-1}\right)^{z-1}} \| \\
\leq \frac{k-\gamma}{2}\left\|u_{n}-u_{n-1}\right\|+\left\|z_{n}-z_{n-1}\right\|+\gamma\left\|U_{n}-u_{n-1}\right\|
\end{gathered}
$$


which implies that

$$
\left\|u_{n}-u_{n-1}\right\| \leq \frac{1}{1-\left(\frac{k+\gamma}{2}\right)}\left\|z_{n}-z_{n-1}\right\| .
$$

Thus, from (3.17) and (3.18), we have

$$
\begin{gathered}
\left\|z_{n+1}-z_{n}\right\| \leq\left\{\frac{\frac{k-\gamma}{2}+\rho \xi \mu+\sqrt{1-2 \rho \alpha+\rho^{2} \beta^{2}}}{1-\frac{1}{2}(k+\gamma)}\right\}\left\|z_{n}-z_{n-1}\right\| \\
=\theta\left\|z_{n}-z_{n-1}\right\|
\end{gathered}
$$

with

$$
\theta=\frac{\frac{k-\gamma}{2}+\rho \xi \mu+\sqrt{1-2 \rho \alpha+\rho^{2} \beta^{2}}}{1-\frac{1}{2}(k+\gamma)} .
$$

Now from (3.9), (3.10), and (3.11), we have $\theta<1$. Hence, from (3.19), it follows that $\left\{z_{n}\right\}$ is a Cauchy sequence in $H$; that is, $z_{n+1} \rightarrow z \in H$, as $n \rightarrow \infty$. From (3.18), we know that $\left\{u_{n}\right\}$ is also a Cauchy sequence in $H$; that is, $u_{n+1} \rightarrow u$ as $n \rightarrow \infty$.

From (3.7), we have

$$
\left\|w_{n+1}-w_{n}\right\| \leq M\left(V\left(u_{n+1}\right), V\left(u_{n}\right)\right) \leq \mu\left\|u_{n+1}-u_{n}\right\|,
$$

from which it follows that $\left\{w_{n}\right\}$ is a Cauchy sequence in $H$; that is, $w_{n+1} \rightarrow w$ as $n \rightarrow \infty$.

Using the continuity of the operators $T, g, A, V, P_{K}$, and Algorithm 3.1, we have

$$
z=g(u-\rho(T u+A w)
$$

that is,

$$
z=P_{K(u)} z-\rho\left(T g^{-1} P_{K(u)} z+A w\right) \in H .
$$

Now we show that $w \in V(u)$. In fact,

$$
\begin{gathered}
d(w, V(u)) \leq\left\|w-w_{n}\right\|+d\left(w_{n}, V(u)\right) \\
\leq\left\|w-w_{n}\right\|+M\left(V\left(u_{n}\right), V(u)\right) \\
\leq\left\|w-w_{n}\right\|+\mu\left\|u_{n}-u\right\|,
\end{gathered}
$$

where $d(w, V(u))=\inf \{\|y-v\|: v \in V(u)\}$. Since the sequences $\left\{u_{n}\right\}$ and $\left\{w_{n}\right\}$ are the Cauchy sequences, it follows from the above inequality that $d(w, V(u))=0$, which implies that $w \in V(u)$, due to $V(u) \in C(H)$. Using Theorem 3.1, we see that $z \in H, u \in H, w \in V(u)$ are solutions of (2.2) and consequently $z_{n+1} \rightarrow z, u \rightarrow u$, and $w_{n+1} \rightarrow w$ strongly in $H$. This completes the proof. 


\section{Auxiliary Principle Technique}

In this section, we use the auxiliary principle technique of Glowinski, Lions, and Tremolieres [10] and Noor [18-23] to prove the existence of a unique solution of the strongly nonlinear mixed variational-like inequality (2.3), which is the prime motivation of our main result.

Theorem 4.1: Let an operator $T: H \rightarrow H$ be strongly monotone with constant $\alpha>0$ and Lipschitz continuous with constant $\beta>0$. Let an operator $\eta: H \times H \rightarrow H$ be strongly monotone with constant $\sigma>0$ and Lipschitz continuous with constant $\delta>0$. Let operator $A: H \rightarrow H$ be Lipschitz continuous with constant $\gamma>0$ and Assumption 2.1 hold. If $k<\alpha$, where $\alpha$ is the strongly monotonicity constant of $T$, and $k$ is a constant as defined by (4.5), then there exists a unique solution $u \in H$ of variational-like inequality (2.3).

Proof: (a) Uniqueness: Let $u_{1}, u_{2} \in H, u_{1} \neq u_{2}$ be two solutions of problem (2.3); that is

$$
\left\langle T u_{1}+A\left(u_{1}\right), \eta\left(v, u_{1}\right)\right\rangle+j(v)-j\left(u_{1}\right) \geq 0 \text { for all } v \in H
$$

and

$$
\left\langle T u_{2}+A\left(u_{2}\right), \eta\left(v, u_{2}\right)\right\rangle+j(v)-j\left(u_{2}\right) \geq 0 \text { for all } v \in H .
$$

Taking $v=u_{2}$ (respectively $u_{1}$ ) in (4.1) (respectively (4.2)), performing the summation of the resultant inequalities and using the assumption that $\eta\left(u_{1}, u_{2}\right)=-\eta\left(u_{2}, u_{1}\right)$, we have

$$
\left\langle T u_{1}-T u_{2}, \eta\left(u_{1}, u_{2}\right)\right\rangle \leq-\left\langle A\left(u_{1}\right)-A\left(u_{2}\right), \eta\left(u_{1}, u_{2}\right)\right\rangle
$$

which can be written as

$$
\left\langle T u_{1}-T u_{2}, u_{1}-u_{2}\right\rangle \leq\left\langle T u_{1}-T u_{2}, u_{1}-u_{2}-\eta\left(u_{1}, u_{2}\right)\right\rangle-\left\langle A\left(u_{1}\right)-A\left(u_{2}\right), \eta\left(u_{1}, u_{2}\right)\right\rangle .
$$

Now using the strong monotonicity and Lipschitz continuity of the operator $T$, we have

$$
\begin{aligned}
\alpha\left\|u_{1}-u_{2}\right\|^{2} \leq & \left\|T u_{1}-T u_{2}\right\|\left\|u_{1}-u_{2}-\eta\left(u_{1}, u_{2}\right)\right\|+\left\|A\left(u_{1}\right)-A\left(u_{2}\right)\right\|\left\|\eta\left(u_{1}, u_{2}\right)\right\| \\
& \leq\left\{\beta\left\|u_{1}-u_{2}-\eta\left(u_{1}, u_{2}\right)\right\|+\gamma\left\|\eta\left(u_{1}, u_{2}\right)\right\|\right\}\left\|u_{1}-u_{2}\right\|
\end{aligned}
$$

where $\gamma>0$ is the Lipschitz continuity constant of $A$.

Since the operator $\eta$ is strongly monotone and Lipschitz continuous, it follows that

$$
\begin{aligned}
\left\|u_{1}-u_{2}-\eta\left(u_{1}, u_{2}\right)\right\|^{2} & =\left\|u_{1}-u_{2}\right\|^{2}-2\left\langle\eta\left(u_{1}, u_{2}\right), u_{1}-u_{2}\right\rangle+\left\|\eta\left(u_{1}, u_{2}\right)\right\|^{2} \\
& \leq\left(1-2 \sigma+\delta^{2}\right)\left\|u_{1}-u_{2}\right\|^{2} .
\end{aligned}
$$

From (4.3), (4.4), and the Lipschitz continuity of $\eta$, we obtain

$$
\alpha\left\|u_{1}-u_{2}\right\|^{2} \leq\left\{\beta\left(\sqrt{1-2 \sigma+\delta^{2}}\right)+\gamma \delta\right\}\left\|u_{1}-u_{2}\right\|^{2}=k\left\|u_{1}-u_{2}\right\|^{2},
$$

where

$$
k=\beta\left(\sqrt{1-2 \sigma+\delta^{2}}\right)+\gamma \delta .
$$


Thus,

$$
(\alpha-k)\left\|u_{1}-u_{2}\right\|^{2} \leq 0
$$

which implies that $u_{1}=u_{2}$, the uniqueness of the solution, since $k<\alpha$.

(b) Existence: We now use the auxiliary principle technique (see Noor [18-23]) to prove the existence of a solution of the mixed variational-like inequality (2.3). For a given $u \in H$, we consider the problem of finding a unique $w \in H$ satisfying the auxiliary mixed variational-like inequality

$$
\langle w, v-w\rangle+\rho j(v)-\rho j(w) \geq\langle u, v-w\rangle-\rho\langle T u+A(u), \eta(v, w)\rangle,
$$

for all $v \in H$, where $\rho>0$ is a constant.

Relation (4.6) defines a mapping $u \rightarrow w$. In order to prove the existence of a solution of (2.3), it is sufficient to show that the mapping $u \rightarrow w$, defined by (4.6), has a fixed point belonging to $H$ which satisfies (2.3). In other words, it is sufficient to show that for a relevant choice of $\rho>0$, $\left\|w_{1}-w_{2}\right\| \leq \theta\left\|u_{1}-u_{2}\right\|$ with $0<\theta<1$, where $\theta$ is independent of $u_{1}$ and $u_{2}$. Let $w_{1}$ and $w_{2}$ be two solutions of (4.6) related to $u_{1}$ and $u_{2}$, respectively. Taking $v=w_{2}$ (respectively $w_{1}$ ) in (4.6) related to $u_{1}$ (respectively $u_{2}$ ), we have that

$$
\left\langle w_{1}, w_{2}-w_{1}\right\rangle+\rho j\left(w_{2}\right)-\rho j\left(w_{1}\right) \geq\left\langle u_{1}, w_{2}-w_{1}\right\rangle-\rho\left\langle T u_{1}+A\left(u_{1}\right), \eta\left(w_{2}, w_{1}\right)\right\rangle
$$

and

$$
\left\langle w_{2}, w_{1}-w_{2}\right\rangle+\rho j\left(w_{1}\right)-\rho j\left(w_{2}\right) \geq\left\langle u_{2}, w_{1}-w_{2}\right\rangle-\rho\left\langle T u_{2}+A\left(u_{2}\right), \eta\left(w_{1}, w_{2}\right)\right\rangle .
$$

Adding these inequalities and using the assumption $\eta\left(w_{1}, w_{2}\right)=-\eta\left(w_{2}, w_{1}\right)$ for all $w_{1}, w_{2} \in H$, we have

$$
\begin{gathered}
\left\langle w_{1}-w_{2}, w_{1}-w_{2}\right\rangle \leq\left\langle u_{1}-u_{2}, w_{1}-w_{2}\right\rangle-\rho\left\langle T u_{1}-T u_{2}, \eta\left(w_{1}, w_{2}\right)\right\rangle-\rho\left\langle A\left(u_{1}\right)-A\left(u_{2}\right), \eta\left(w_{1}, w_{2}\right)\right\rangle \\
=\left\langle u_{1}-u_{2}-\rho\left(T u_{1}-T u_{2}\right), w_{1}-w_{2}\right\rangle+\rho\left\langle T u_{1}-T u_{2}, w_{1}-w_{2}-\eta\left(w_{1}, w_{2}\right)\right\rangle \\
-\rho\left\langle A\left(u_{1}\right)-A\left(u_{2}\right), \eta\left(w_{1}, w_{2}\right)\right\rangle
\end{gathered}
$$

from which it follows that

$$
\begin{gathered}
\left\|w_{1}-w_{2}\right\|^{2} \leq\left\|u_{1}-u_{2}-\rho\left(T u_{1}-T u_{2}\right)\right\|\left\|w_{1}-w_{2}\right\| \\
+\rho\left\|T u_{1}-T u_{2}\right\|\left\|w_{1}-w_{2}-\eta\left(w_{1}, w_{2}\right)\right\| \\
+\rho\left\|A\left(u_{1}\right)-A\left(u_{2}\right)\right\|\left\|\eta\left(w_{1}, w_{2}\right)\right\| .
\end{gathered}
$$

Combining (4.4), (4.9), (3.14), and using the Lipschitz continuity of $T, A$, and $\eta$, we have

$$
\begin{aligned}
\left\|w_{1}-w_{2}\right\| \leq & \left\{\sqrt{1-2 \rho \alpha+\rho^{2} \beta^{2}}+\rho\left(\beta \sqrt{1-2 \sigma+\delta^{2}}+\delta \gamma\right)\right\}\left\|u_{1}-u_{2}\right\| \\
& =\{t(\rho)+\rho k\}\left\|u_{1}-u_{2}\right\|=\theta\left\|u_{1}-u_{2}\right\|,
\end{aligned}
$$

where $\theta=t(\rho)+\rho k, t(\rho)=\sqrt{1-2 \rho \alpha+\beta^{2} \rho^{2}}$ and $k=\beta \sqrt{1-2 \sigma+\delta^{2}}+\delta \gamma$. 
We need to show that $\theta<1$. It is clear that $t(\rho)$ reaches its minimum value at $\bar{\rho}=\frac{\alpha}{\beta^{2}}$ with $t(\bar{\rho})=\sqrt{1-\frac{\alpha^{2}}{\beta^{2}}}$. For $\rho=\bar{\rho}, \rho k+t(\rho)<1$ implies that $\rho k<1$. Thus, it follows that $\theta<1$ for all $\rho$ with $0<\rho<2 \frac{\alpha-k}{\beta^{2}-k^{2}}, \rho k<1$ and $k<\alpha$. Consequently, the mapping $u \rightarrow w$ defined by (4.6) has a fixed point belonging to $H$, which is a solution of the mixed variational-like inequality (2.3). This completes the proof.

Remark 4.1: If $\eta(v, u)=v-u$, then problem (4.6) is equivalent to finding $w \in H$ for a given $u \in H$ such that

$$
\langle w, v-w\rangle+\rho j(v)-\rho j(u) \geq\langle u, v-w\rangle-\rho\langle T u+A(u), v-w\rangle, \text { for all } v \in H .
$$

From the proof of Theorem 4.1, we see that $k=\gamma$ and $\theta=t(\rho)+\rho \gamma<1$ for $0<\rho<2 \frac{\alpha-\gamma}{\beta^{2}-\gamma^{2}}$, $\gamma<\alpha$, and $\rho \gamma<1$, so that the mapping $u \rightarrow w$ defined by (4.10) has a fixed point, which is the solution of the variational inequality (2.4) studied by Noor [16] in elasticity and Bingham fluid (in 1975).

Remark 4.2: We note that if $w=u$, then $u$ is a solution of the mixed variational-like inequality (2.3). This observation enables us to suggest the following novel iterative algorithm, which is a useful way of computing a solution of (2.3) as long as (4.6) is easier to solve than (2.3).

General Algorithm 4.1:

(a) At $n=0$, start with the initial value $w_{0}$.

(b) At step $n$, solve the auxiliary problem (4.6) with $u=w_{n}$. Let $w_{n+1}$ denote the solution of the problem (4.6).

(c) For a given $\epsilon>0$, if $\left\|w_{n+1}-w_{n}\right\| \leq \epsilon$ stop. Otherwise go back to $(b)$.

Remark 4.3: It is worth mentioning that many previously known methods, including projection techniques and its variant forms, linear approximation, relaxation, descent, and Newton's algorithms, that have been proposed for solving various classes of variational inequalities, and complementarity problems, can be derived as special cases of the auxiliary principle technique; see Zhu and Marcotte [35] and Noor [24, 23]. It has been shown in Noor [23] that the auxiliary principle technique can be used to formulate the equivalent differentiable optimization problems for variational inequalities. We note that the auxiliary principle technique is quite general, flexible, and provides us with a unified framework for computing an approximate solution of variational inequalities. By a suitable choice of the auxiliary problem, one can suggest a number of equivalent formulations for various types of the variational inequalities, and one can also study the existence of the solution of the variational inequalities.

The auxiliary principle technique can be modified and extended for variational inequalities problems involving the multivalued and fuzzy operators. We note that if the convex set involved also implicitly or explicitly depends on the solution, then problem (2.3) is called the generalized mixed quasi-variational-like inequality problem. An extension of the auxiliary principle technique is still an open problem and this constitutes an important and interesting area of future research. Development of efficient and computable iterative algorithms for numerically solving the variational-like inequalities needs further research efforts.

\section{References}

[1] Baiocchi, C. and Capelo, A., Variational and Quasi-Variational Inequalities, J. Wiley and Sons, London and New York 1984. 
[2] Bensoussan, A. and Lions, J.L., Applications des Inequations Variationelles en Constrol et en Stochastiques, Dunod, Paris 1978.

[3] Blum, E. and Oettli, W., From optimization and variational inequalities to equilibrium problems, Math. Student 63 (1994), 123-145.

[4] Chang, S. and Huang, N.J., Generalized strongly nonlinear quasi-complementarity problems in Hilbert spaces, J. Math. Anal. Appl. 158 (1991), 194-202.

[5] Cohen, G., Auxiliary problem principle extended to variational inequalities, J. Optim. Theory Appl. 59 (1988), 325-333.

[6] Cottle, R.W., Pang, J.S., and Stone, R.E., The Linear Complementarity Problem, Academic Press, New York 1992.

[7] Crank, J., Free and Moving Boundary Problems, Clarendon Press, Oxford, UK 1984.

[8] Dafermos, S., Exchange price equilibrium and variational inequalities, Math. Programming 46 (1990), 391-402.

[9] Fichera, G., Problemi elastostatici con vincoli unilaterali: il problema di Signorini con ambigue condizione al contorno, Att Accad. Naz. Lincei Mem. Cl. Sci. Fis. Mat. Natur Sez. Ia 7 (8) (1963-64), 91-140.

[10] Glowinski, R.F., Lions, J.L., and Tremolieres, R., Numerical Analysis of Variational Inequalities, North-Holland, Amsterdam 1981.

[11] Harker, P.T. and Pang, J.S., Finite dimensional variational inequality and nonlinear complementarity problems: a survey of theory, algorithms and applications, Math. Programming 48 (1990), 161-220.

[12] Jou, C.R. and Yao, Y.C., Algorithms for generalized multivalued variational inequalities in Hilbert spaces, Computers Math. Applic. 25 (9) (1993), 7-16.

[13] Kravvarits, D., Nonlinear equations and inequalities in Banach spaces, J. Math. Anal. Appl. 67 (1979), 205-214.

[14] Larsson, T. and Patriksson, M., A class of gap functions for variational inequalities, Math. Program 64 (1994), 53-79.

[15] Noor, M. Aslam, An iterative algorithm for variational inequalities, J. Math. Anal. Appl. 158 (1991), 448-455.

[16] Noor, M. Aslam, On Variational Inequalities, Ph.D. Thesis, Brunel University, London, UK 1975.

[17] Noor, M. Aslam, Iterative algorithms for nonlinear variational inequalities, PanAmer. Math. J. 3:2 (1993), 61-80.

[18] Noor, M. Aslam, General nonlinear variational inequalities, J. Math. Anal. Appl. 128 (1987), 78-84.

[19] Noor, M. Aslam, Variational-like inequalities, Optimization 30 (1994), 323-330.

[20] Noor, M. Aslam, General auxiliary principle technique for variational inequalities, PanAmer. Math. J. 4 (1) (1994), 27-44.

[21] Noor, M. Aslam, Nonconvex functions and variational inequalities, J. Optim. Theory Appl. 87 (1995), (in press).

[22] Noor, M. Aslam, Nonlinear variational inequalities in elastostatics, Internat. J. Eng. Sci. 26 (1988), 1043-1051.

[23] Noor, M. Aslam, Equivalence of differentiable optimization problems for variational inequalities, J. Nat. Geometry 8 (1995), 117-128.

[24] Noor, M. Aslam, Variational inequalities in physical oceanography, In: Ocean Waves Engineering (edited by M. Rahman), Comput. Mechanics Publ., UK (1994), 201-226.

[25] Noor, M. Aslam, Multivalued general quasi-variational inequalities, Nonlinear World 2 (1995), (in press).

[26] Noor, M. Aslam, Set-valued variational inequalities, Optimization 33 (1995), 133-142.

[27] Noor, M. Aslam, Noor, K. Inayat, and Rassias, Th.M., Some aspects of variational inequalities, J. Comput. Appl. Math. 47 (1993), 285-312. 
[28] Noor, M. Aslam, Noor, K. Inayat, and Rassias, Th.M., Invitation to variational inequalities, In: Analysis, Geometry and Groups: A Riemann Legacy Volume (edited by H.M. Srivastava and Th.M. Rassias), Hadronic, USA (1993), 373-448.

[29] Noor, M. Aslam and Oettli, W., On general nonlinear complementarity problems and quasi-equilibria, Le Matematiche 49 (1994), 373-448.

[30] Shi, P., Equivalence of variational inequalities with Wiener-Hopf equations, Proc. Amer. Math. Soc. 111 (1991), 339-346.

[31] Speck, F.O., General Wiener-Hopf Factorization Methods, Pitman Res. Notes Math. Set. No. 119, Longman, Harlow, UK 1985.

[32] Stampacchia, G., Formes bilineaires coercitives sur les ensembles convexes, C.R. Aca. Sci. Paris, Sér I. Math. 258 (1964), 4413-4416.

[33] Yang, X.Q. and Chen, G.Y., A class of nonconvex functions and pre-variational inequalities, J. Math. Anal. Appl. 169 (1992), 359-373.

[34] Yao, J.C., The generalized quasi-variational inequality problem with applications, J. Math. Anal. Appl. 158 (1991), 139-160.

[35] Zhu, D. and Marcotte, P., An extended descent framework for variational inequalities, $J$. Optim. Theory Appl. 80 (1994), 340-366. 


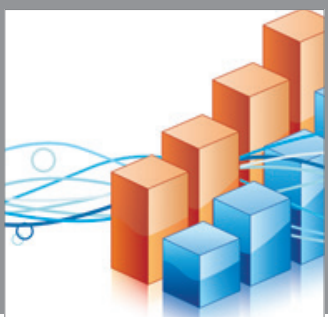

Advances in

Operations Research

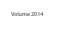

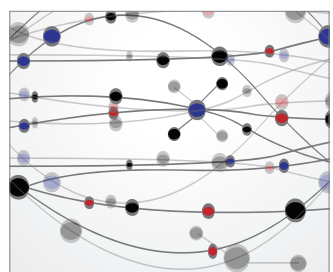

\section{The Scientific} World Journal
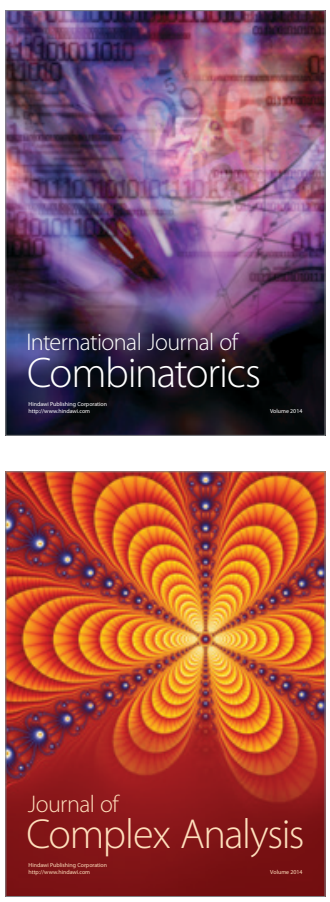

International Journal of

Mathematics and

Mathematical

Sciences
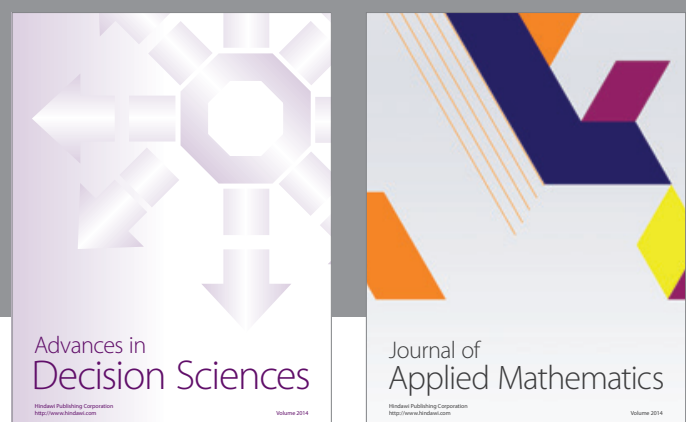

Journal of

Applied Mathematics
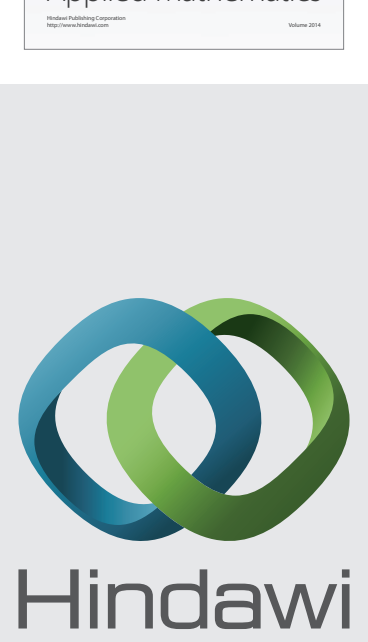

Submit your manuscripts at http://www.hindawi.com
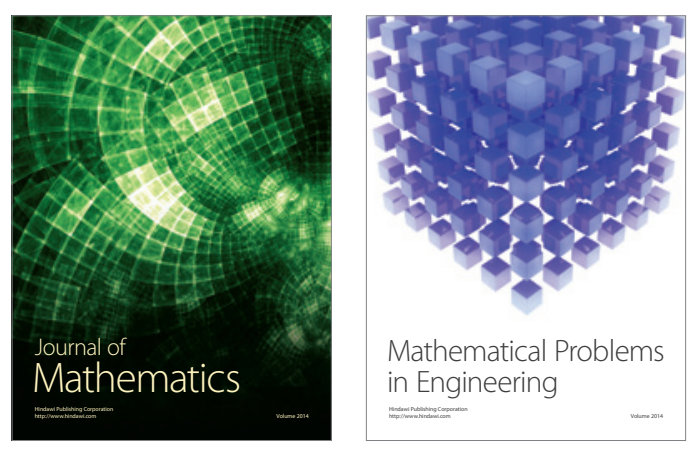

Mathematical Problems in Engineering
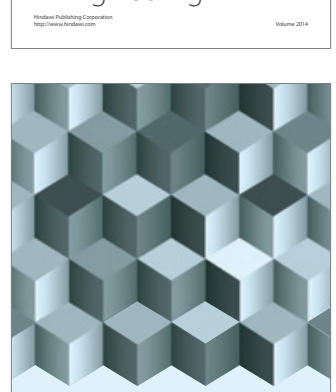

Journal of

Function Spaces
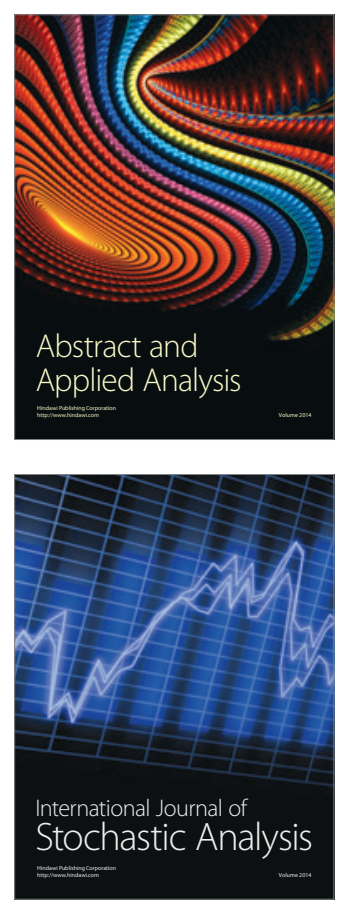

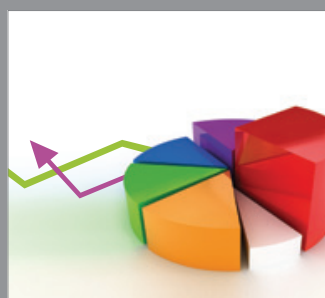

ournal of

Probability and Statistics

Promensencen
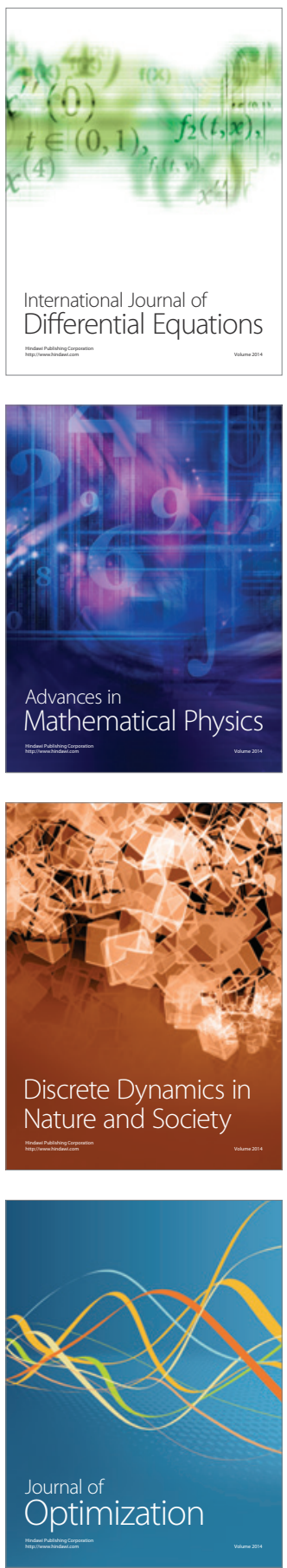